\title{
User modelling and adaptive, natural interaction for conflict resolution
}

\author{
Kostas \\ Karpouzis \\ Image, Video and \\ Multimedia \\ Systems Lab, \\ NTUA, Greece
}

\author{
George \\ Yannakakis \\ Centre for Comp. \\ Games Research \\ IT University, \\ Denmark
}

\author{
Ana Paiva \\ Intelligent Agents \\ and Synthetic \\ Characters Group, \\ INESC-ID, \\ Lisboa, Portugal
}

\author{
Jeppe Herlev \\ Nielsen \\ Serious Games \\ Interactive, \\ Denmark
}

\author{
Asimina \\ Vasalou \\ HCI Centre \\ University of \\ Birmingham, \\ UK
}

\author{
Arnav Jhala \\ Baskin School of \\ Engineering \\ University of \\ California Santa \\ Cruz, USA
}

\begin{abstract}
Confronting conflicts and coping with them is part of social life, since conflicts seem to arise in almost every context and developmental stage of human life. The personal and collective gains that follow conflict resolution have motivated scholars across many research fields to advocate the use of pro-social mechanisms for resolution. The Siren serious game aims to support teachers' role to educate young people on how to resolve conflicts, by employing affect-aware, userand cultural adaptivity to provide interesting and relevant conflict scenarios and resolution approaches.
\end{abstract}

Keywords: serious games, conflict resolution, user modelling, cross-cultural user studies, affective interaction, adaptation

\section{INTRODUCTION}

Confronting conflicts and coping with them is part of social life. Indeed, conflicts seem to arise in almost every context and developmental stage of human life, from scuffles in schoolyards, to bullying in the workplace and to international warfare. The personal and collective gains that follow conflict resolution have motivated scholars in the fields of law, education, organisational management, psychology and social science, among others, to advocate the use of pro-social mechanisms for resolution. Interventions that may impart individuals with experience in resolving conflicts will be of clear benefit to society.

A complex web of societal structures, technological advances and developmental processes call for immediate action to the problem of conflicts. On societal level, as European countries become increasingly multi-cultural and ethnically diverse, integration has become more problematic due to clusters of cultural and ethnic populations that naturally form in major urban centres. Clashing social dynamics often emerge in these areas, which in turn have flow-on effects on the wider European social dynamics. On a technological level, the advances of modern society, pose new dangers for an already vulnerable population, children, since not only are young people experiencing the intrusion of unresolved conflicts in their private spaces (e.g. via the Internet or the mobile phone), but also their perception of harm is magnified in the context of a youth culture that insatiately seeks the acceptance of the peer group. On a developmental level, entering the educational environment is daunting for some children. The classroom is the stage where learning difficulties (e.g. dyslexia), anti-social and deviant behaviour are enacted. Although family dynamics can be the root of many behavioural problems [9], the educational system is responsible for reinstating children that suffer from these difficulties and giving them the tools they need to manage their individual needs.

Serious games have already proven viable and effective for promoting intercultural communication and representing different perspectives on social issues. Almost invariably, computer games contain elements of conflict, often between the character controlled by the player and either one or several non-player characters (NPCs), or between characters controlled by a group of human players. In many, though not all, contemporary computer games, violence is the most common method for resolving conflict. To win the game entails that other players or characters lose. In opposition to these competitive and retributive models of computer games, engaging and entertaining games whose objective is to overcome obstacles in collaboration with other players in a non-violent way are gaining status. Instead of rewarding and reinforcing competitive behaviour, this genre of games reward winning by means of collaborative, non-violent problem solving.

\section{THE SIREN GAME}

The main aim of the Siren game (http://sirenproject.eu) is to teach peaceful and constructive ways for resolving conflicts: through perspective taking, kids will learn to negotiate as opposed to avoid or retaliate during conflicts. The players (who can be divided into one or several groups or "sides", "tribes" or "theatre companies") will face a conflict situation together. Examples of domains are a classroom theatre club, an after-school sporting event and a village/farm environment. In this framework, mini-games in the context of the theatre game present friendship disputes, while the village game present several forms of property dispute or resource management. In terms of game mechanics, these kinds of scenarios can be formalised as collaborative puzzle solving with constraints, where each participant has incomplete information about the overall state of the game. All of these elements support the learning objectives of the game by immersing players in the conflict, facilitating a critical approach to their assumptions about the conflict and allowing them to explore new perspectives.

The process of identifying relevant conflict situations for the targeted user group (10-11 y.o. children) included interviews about conflicts, how they begin, escalate and are resolved, emotions related to each phase and possible interventions during this process. In addition to this, a series of cultural probes [4] were used to quantify that information and also identify possible social dynamics and cross-cultural differences in the UK, Portugal and Greece. Interviews took place in two schools in each country (ca. 30 children in each 
school), one of which was in a middle/working class region, while the other was a private or experimental school. The aims of this user study were to identify what constitutes conflict in terms of quantitative terms and to define a cultural model, which can be compared across countries and schools. In the fields of anthropology, sociology, and cross-cultural psychology, culture has long been acknowledged as a fundamental and crucial factor in how people perceive the world, and how they behave. Culture has been defined by Hofstede as the "collective programming of the mind which distinguishes the members of one group or category of people from another" [5]. Within the simulation literature, games have frequently been used to acclimatise people towards understanding cultural differences (e.g. [3], [7], [8])

\section{A. Concepts of affect and adaptation}

One of the main innovations of Siren is the ability to produce adaptive game scenarios regarding both the gaming experience and the conflicts mini game scenarios. In the first case, we employ affect and attention detection techniques, based on the video feed from a plain web camera mounted on top of the player's screen, to estimate the player's emotional and cognitive state [1]. The detected state, associated with in-game behaviour and performance, is then used to place the user on the 2D map of "Flow" [2]; the assumption here is that when the player is in the state of Flow, i.e. fully immersed in what he or she is doing, the game becomes utterly interesting and teaching the learning objectives of the game becomes intuitive.

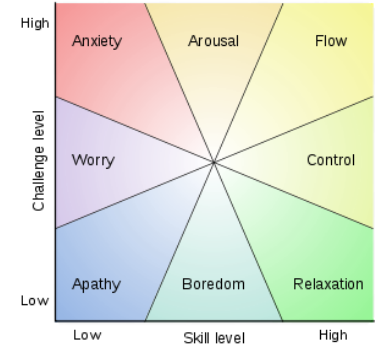

Figure 1: Mental state vs. challenge \& skill level [2]

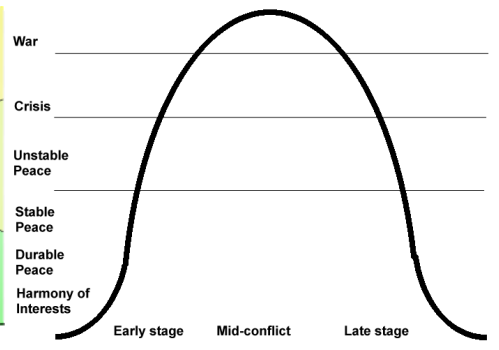

Figure 2: The Conflict Cycle
The axes in Figure 1 correspond to the skill level of the player and the challenge level posed by a particular mini game instance to that player: if the visual analysis procedure estimates that the user is "bored" (thereby inattentive [1]), the Siren game produces a harder instance of the mini-game (e.g. less resource with which to get by), attempting to match the user's skill level and make the game more interesting. Similarly, the affective analysis component tracks the user's facial expression in order to detect the point of the conflict cycle the user is at a given time and, thus, estimate their progress towards completion of the particular mini-game. Definition of relevant facial expressions and head pose thresholds to denote user attention was based on annotating and analysing the Siren gameplay database, one of the few visual affect databases focusing on gameplay, available from the project website ([12]). Figure 2 illustrates a conflict cycle, characterised by an escalation phase followed by a deescalation phase [11]. Again, based on affective, cognitive and behavioural information from the user, we estimate the stage in the conflict cycle that the player is in, which is a measure of the player's progress in the game. This caters for procedurally generating events in the particular mini-game, helping the player successfully resolve the conflict. After completion of the aims of the particular mini-game, the user's model is adapted based on affective and behavioural indicators, producing a relevant and interesting instance of the next mini-game. To facilitate this process, mini games are procedurally generated [10], based on parameters mapped to predicted levels of challenge and frustration [12].

\section{CONCLUSIONS - FUTURE WORK}

The Siren project aims to produce an adaptive game to teach productive conflict strategies via popular game themes, taking into account emerging non-verbal information from the part of the user, after conducting extensive user studies have been to facilitate scenario development and user modelling. During the next year, we plan evaluate a set of playable mini-games in schools in three different European countries early enough to inform the design and development processes with user feedback.

\section{ACKNOWLEDGEMENT}

This work has been partially funded by the DG INFSO Project SIREN (258453) Technology Enhanced Learning.

\section{REFERENCES}

[1] S. Asteriadis, P. Tzouveli, K. Karpouzis, S. Kollias, Estimation of behavioral user state based on eye gaze and head pose, Multimedia Tools and Applications, Springer, 41 (3), pp. 469-493, 2009.

[2] M. Csikszentmihalyi, Flow: The Psychology of Optimal Experience, New York: Harper and Row, 1990.

[3] S. Fowler, M. Pusch, Intercultural Simulation Games: A Review (of the United States and Beyond), Simulation and Gaming, 2010, 41; 94.

[4] B. Gaver, T. Dunne, E. Pacenti, Design: Cultural probes, interactions, 6, 1, 21-29, 1999.

[5] G. Hofstede, Cultures and Organizations: Software of the Mind: Intercultural Cooperation and Its Importance for Survival, McGrawHill: New York, 1996.

[6] S. Ioannou, A. Raouzaiou, V. Tzouvaras, T. Mailis, K. Karpouzis, S. Kollias, Emotion recognition through facial expression analysis based on a neurofuzzy network, Neural Networks, Elsevier, 18 (4), 423-435.

[7] G. Hofstede, P. Pedersen, Synthetic Cultures: Intercultural Learning Through Simulation Games, Simulation and Gaming, 1999; 30; 415.

[8] R. Khaled, P. Barr, R. Biddle, R. Fischer, J. Noble, Game design strategies for collectivist persuasion. In Proceedings of the 2009 ACM SIGGRAPH Symposium on Video Games, New York, 31-38.

[9] M. Margalit and K. Almougy, Classroom behavior and family climate in students, with learning disabilities and hyperactive behavior, Journal of Learning Disabilities, 24, 406-412, 1991.

[10] G. Yannakakis, J. Togelius, Experience-driven Procedural Content Generation, IEEE Trans. Affective Computing, 2(3), 147-161, 2011.

[11] N. Swanstrom, M. Weissmann. Conflict, Conflict Prevention and Conflict Management and Beyond: A Conceptual Exploration, Central Asia-Caucasus Inst. \& Silk Road Studies Programme, 2005.

[12] S. Asteriadis, N. Shaker, K. Karpouzis, G. N. Yannakakis, Towards Player's Affective and Behavioral Visual Cues as drives to Game Adaptation, LREC Workshop on Multimodal Corpora, Istanbul 2012. 